\title{
Acupuntura na prática odontológica: Ênfase na cirurgia
}

\author{
Acupuncture in dental practice: Emphasis in surgery \\ Acupuntura en la práctica dental: Énfasis en la cirugía
}

Recebido: 22/03/2021 | Revisado: 30/03/2021 | Aceito: 03/04/2021 | Publicado: 13/04/2021

\author{
Ana Luisa Ferreira Lemos \\ ORCID: https://orcid.org/0000-0001-9680-6487 \\ Faculdade Patos de Minas, Brasil \\ E-mail: analuisa_fl@hotmail.com \\ Amanda Sousa Teixeira \\ ORCID: https://orcid.org/0000-0001-5989-4158 \\ Faculdade Patos de Minas, Brasil \\ E-mail: amanda.sousa.tx@gmail.com \\ Fernando Nascimento \\ ORCID: https://orcid.org/0000-0003-3568-3887 \\ Faculdade Patos de Minas, Brasil \\ E-mail: fernando.nascimento@ faculdadepatosdeminas.edu.br \\ Luciana de Araújo Mendes Silva \\ ORCID: https://orcid.org/0000-0003-0311-1323 \\ Faculdade Patos de Minas, Brasil \\ E-mail: laraujo32016@gmail.com \\ Marcelo Dias Moreira de Assis Costa \\ ORCID: https://orcid.org/0000-0001-9148-3674 \\ Universidade Federal de Uberlândia, Brasil \\ E-mail: marcelodmac@yahoo.com.br \\ Lia Dietrich \\ ORCID: https://orcid.org/0000-0001-7887-8591 \\ Clínica privada, Brasil \\ E-mail: dietrichlia.ld@gmail.com
}

\begin{abstract}
Resumo
Atualmente, existe uma grande busca por terapias consideradas naturais, geralmente chamadas de alternativas, tanto por parte dos cirurgiões dentistas, quanto dos pacientes. Em meio a diversas opções, encontra-se a acupuntura, uma técnica que faz parte da Medicina Tradicional Chinesa, a qual, se constitui pela inserção de agulhas, em pontos específicos da pele, ao longo do corpo. Tal técnica comprovou cientificamente suas capacidades ansiolíticas, hemostáticas, anti-inflamatórias, analgésicas, mio relaxantes e ativadora da função imune. Este trabalho objetiva agrupar informações, sobre o benefício da acupuntura dentro da odontologia, dando enfoque aos procedimentos cirúrgicos. Foi realizada uma revisão de literatura narrativa do tema, utilizando como base de referência artigos disponíveis em plataformas onlines, como BVS salud, Scielo e Pubmed, nos idiomas inglês e portugues (Brasil), onde foi priorizado a utilização de artigos mais atuais ou artigos que trazem a base e fundamentação da acupuntura. A acupuntura se mostra uma alternativa, extremamente interessante, tanto para o paciente quanto para o cirurgião dentista, uma vez que, proporciona segurança e conforto para ambos.
\end{abstract}

Palavras-chave: Odontologia; Analgesia por acupuntura; Terapia por acupuntura; Acupuntura; Cirurgia bucal.

\begin{abstract}
Currently, there is a great search for alternative therapies, both by dentists and patients. In the midst of several options, there is acupuncture, a technique that is part of Traditional Chinese Medicine, which consists of the insertion of needles, in specific points of the skin, along the body. This technique has scientifically proven its capacities, anxiolytic, hemostatic, anti-inflammatory, analgesic, myo-relaxing and activating the immune function. It is proving to be an extremely interesting alternative for both the patient and the dental surgeon, since it provides safety and comfort for both. This work aims to group information on the benefit of acupuncture within dentistry and expose it in a didactic way to professionals in this area, thus expanding their horizon of knowledge and enabling them to offer this alternative to patients and increasing thus the range of options for these. A narrative literature review of the theme was carried out, using as a reference base 20 articles available on online platforms, such as VHL salud, Scielo and Pubmed. Articles in Portuguese and English were used.
\end{abstract}

Keywords: Dentistry; Acupuncture analgesia; Acupuncture therapy; Acupuncture; Surgery oral. 


\begin{abstract}
Resumen
Actualmente, existe una gran búsqueda de terapias consideradas naturales, generalmente llamadas alternativas, tanto por parte de dentistas como de pacientes. En medio de varias opciones, está la acupuntura, técnica que forma parte de la Medicina Tradicional China, que consiste en la inserción de agujas, en puntos específicos de la piel, a lo largo del cuerpo. Esta técnica ha demostrado científicamente sus capacidades ansiolíticas, hemostáticas, antiinflamatorias, analgésicas, mio-relajantes e inmunoactivadoras. Este trabajo tiene como objetivo agrupar información sobre el beneficio de la acupuntura dentro de la odontología, centrándose en los procedimientos quirúrgicos. Se realizó una revisión de la literatura narrativa sobre el tema, utilizando como base de referencia artículos disponibles en plataformas en línea, como BVS salud, Scielo y Pubmed, en inglés y portugués (Brasil), donde se utilizó artículos o artículos más actuales. que aportan la base y el fundamento de la acupuntura. La acupuntura resulta ser una alternativa sumamente interesante tanto para el paciente como para el dentista, ya que brinda seguridad y comodidad para ambos.
\end{abstract}

Palabras clave: Odontología; Analgesia por acupuntura; Tratamiento con acupuntura; Acupuntura; Cirugía oral.

\title{
1. Introdução
}

A dor é considerada cientificamente como uma resposta protetora intrínseca do próprio organismo contra estímulos que o mesmo considera nocivos para si, o que por sua vez acaba resultando em efeitos indesejáveis uma vez que esta não seja devidamente controlada (Alvarenga, Amaral, \& Steffen, 2014; Boleta-Ceranto \& Miura, 2013). Entretanto apesar de à primeira vista a dor parecer algo negativo, está se trata de um mecanismo extremamente importante para a sobrevivência de todos os seres vivos, a compreensão da dor é um dos mais antigos desejos do ser humano (Alvarenga et al., 2014; Boleta-Ceranto \& Miura, 2013).

Dentro da odontologia, o processo de dor vem sendo classificado como odontogênico e não odontogênico (BoletaCeranto \& Miura, 2013). As dores odontogênicas estão relacionadas aos tecidos dentários e suas respectivas estruturas de suporte, já as dores não odontogênicas são relacionadas aos tecidos mais profundos como os ossos, articulações e/ou músculos (Boleta-Ceranto \& Miura, 2013).

As dores de origem odontogênicas normalmente estão relacionadas a patologias periodontais ou periapicais e em sua grande maioria se apresentam agudas e fáceis de serem diagnosticadas e tratadas (Boleta-Ceranto \& Miura, 2013).

No entanto as dores de origem não odontogênicas em especial aquelas originadas de tecidos profundos são mais graves em relação ao tratamento devido a maior dificuldade de se obter um diagnóstico definitivo (Boleta-Ceranto \& Miura, 2013).

A realização de cirurgias para a exodontias de terceiros molares mandibulares e maxilares é considerado um procedimento extremamente frequente para os cirurgiões dentistas especialista em cirurgia oral (Armond, Glória, Santos, Galo, \& Falci, 2019; Chiapasco, Cicco, \& Marrone, 1993; Gil et al., 2020; Santos et al., 2014).

Existem diversas indicações que levam o cirurgião dentista a diagnosticar a necessidade de realizar a exodontia dos terceiros molares, entre as mais diversas indicações para este procedimento as mais comuns incluem a presença de cáries, pericoronarite, cistos, problemas periodontais e indicações ortodônticas (Armond et al., 2019; Chiapasco et al., 1993; Gil, 2019).

Embora este seja considerado um procedimento extremamente comum dentro dos consultórios odontológicos, o mesmo é constantemente associado a uma serie de complicações pós operatórias, em sua grande maioria associadas ao resultante processo de resposta inflamatória tais como a dor, edemas e trismos (Armond et al., 2019; Gil et al., 2020; SantanaSantos et al., 2013; Santos et al., 2014).

Diversos estudos indicam que a vida profissional bem como sua vida social e sua qualidade de vida sejam diretamente afetas devido o pós-operatório das cirurgias de extração dos terceiros molares (Armond et al., 2019; Santos et al., 2014).

Um estudo realizado na Noruega concluiu que $30 \%$ dos pacientes, que se submeteram a cirurgias para a exodontia de terceiros molares, foram prejudicados em suas rotinas no trabalho e/ou escolas necessitando faltar devido a complicações pós operatórias, sendo que 23,5\% relataram que foram prejudicados devido a dor e 9,5\% devido ao edema (Armond et al., 2019). 
Além dos problemas pós-operatórios, a ansiedade é um estado emocional o qual é constantemente relacionado a cirurgia de extração de terceiros molares (Armond et al., 2019; Gil, 2019). Tal estado pode ocorrer antes ou após o ato cirúrgico, o que por sua vez acaba afetando o paciente tanto psicologicamente como psicossomicamente o que pode acarretar complicações pós-operatórias (Armond et al., 2019; Michalek-Sauberer, Gusenleitner, Gleiss, Tepper, \& Deusch, 2012).

A ansiedade pode variar desde o medo da dor até fobias, os pacientes podem apresenta uma serie de sintomas, tais como tremores e arritmias cardíacas o que em alguns casos acabam impossibilitando a realização do procedimento cirúrgico (Armond et al., 2019; Michalek-Sauberer et al., 2012). Diversos medicamentos podem ser utilizados para reduzir e controlar as complicações pós-operatórias, entre eles estão presentes os anti-inflamatórios não esteroidais, corticosteroides e analgésicos, ansiolíticos, opioides e barbitúricos (Armond et al., 2019; Michalek-Sauberer et al., 2012; Zotelli, Meirelles, \& Sousa, 2010).

Porem esses fármacos apresentam uma serie de efeitos colaterais, o quais podem ser extremamente perigosos para os pacientes, tal fato faz com que tanto os profissionais de saúde quanto os pacientes procurem por opções alternativas para evitarem a ocorrência de tais efeitos adversos (Armond et al., 2019; Lux, Taffarel \& Freitas, 2017; Wahl, Erlenwein, Wiese. \& Wirz, 2017).

Devido as constantes busca por novos tratamentos, a odontologia vem preconizando técnicas alternativas, para auxiliar os profissionais e proporcionar mais conforto e segurança para os pacientes (Boleta-Ceranto \& Miura, 2013; Armond et al., 2019). Quando se fala em novas técnicas, não nos referimos apenas ao desenvolvimento de novos aparelhos ultramodernos ou mesmo fármacos de última geração, diversos estudos demonstram e comprovam, que técnicas milenares se apresentam cientificamente eficientes e dentre essas diversas técnicas destaca-se a acupuntura, que vem sendo a opção de escolha de profissionais e pacientes como método auxiliar que ajuda no alivio da dor e que por sua vez acaba reduzindo a quantidade de fármacos consumidos nos pós-operatórios e assim reduz a quantidade de efeitos colaterais aos quais o paciente é exposto (Boleta-Ceranto \& Miura, 2013; Armond et al., 2019; Lux et al., 2017).

A acupuntura não se restringe exclusiva a um único uso dentro da odontologia, ela vem sendo utilizada em todo o mundo para diversos tratamentos dentro desta área, tais como tratamento de disfunções temporomandibulares, dores orofaciais, auxiliar a técnica anestésica entre outros diversos usos dentro da área odontológica os quais serão citados neste trabalho (Sousa, 2018).

Este trabalho objetiva agrupar informações, sobre o benefício da acupuntura dentro da odontologia e expô-las de forma didática para os profissionais desta área, dando enfoque aos procedimentos cirúrgicos. Ampliando, assim o horizonte de conhecimento destes e possibilitando que os mesmos possam oferecer mais essa alternativa para os pacientes, aumentando desta forma o leque de opções.

\section{Metodologia}

Trata-se de uma pesquisa qualitativa realizada na forma de revisão narrativa de literatura. Conforme Pereira, Shitsuka, Parreira e Shitsuka (2018) em uma pesquisa qualitativa as informações colhidas de forma geral são descritivas e a análise dos dados em geral tende ao método indutivo. Já Günther (2006) menciona que a pesquisa qualitativa origina textos com diferentes técnicas analíticas e nesse sentido não se preocupa com questões de quantidade mas de qualidade das informações.

Uma revisão narrativa da literatura por sua vez, caracteriza-se pela sua busca de descrever determinado assunto do ponto de vista conceitual ou teórico e procura verificar as lacunas existentes na literatura bem como trazer informações que despertem a realização de novas pesquisas (Brum et al., 2015).

Diante dessa escolha metodológica, a pesquisa foi efetivada utilizando-se como base de referência artigos disponíveis em plataformas onlines, como BVS salud, Scielo e Pubmed, bem como dissertações e teses obtidos em bibliotecas digitais, sendo selecionadas obras nos idiomas inglês e portuguÊs (Brasil). Foi priorizada a utilização de fontes mais atuais ou que 
trazem a base e fundamentação da acupuntura.

\section{Revisão da Literatura}

Devido a conscientização a respeito da relação existente entre mente e corpo vem ocorrendo um grande aumento na procura do estabelecimento de um equilíbrio entre estes, para tal vem ocorrendo uma grande busca por tratamentos alternativos para auxiliarem e ampliarem o potencial das terapias convencionais, tal procura acaba forçando o surgimento de novas terapias (Boleta-Ceranto \& Miura, 2013).

A acupuntura é uma prática milenar a qual possui como base o equilíbrio entre mente e corpo (Alvarenga et al., 2014; Boleta-Ceranto \& Miura, 2013; Florian, Rando-Meirelles \& Sousa, 2012; Gil, 2013).

Tal técnica demonstra excelentes resultados em aproximadamente 70 a 80\% dos pacientes (Boleta-Ceranto \& Miura, 2013). Segunda a Food and Drugs Administration (FDA) aproximadamente 9 a 12 milhões de norte-americanos realizam terapias com acupuntura anualmente (Boleta-Ceranto \& Miura, 2013). Estudos alemães demonstram que $90 \%$ dos pacientes entrevistados afirmam que a terapia com a acupuntura conseguiu aliviar suas dores (Alvarenga et al., 2014; Boleta-Ceranto \& Miura, 2013).

O nome acupuntura vem do latim acus = agulha e punctura $=$ picada, portanto logo refere-se à introdução de agulhas em pontos específicos da pele, pontos esses chamados de acupontos os quais foram determinados de forma empírica ao transcorrer de milhares de ano de prática médica, tal procedimento objetiva originalmente a cura e prevenção de males, a acupuntura também é conhecida como uma terapêutica reflexiva, uma vez que ao estimular um ponto do corpo outro ponto sofre uma reação, utilizando-se principalmente do estimulo nociceptivo (Alvarenga et al., 2014; Arslan et al., 2019; BoletaCeranto \& Miura, 2013; Grillo, 2011; Sousa, 2014, ).

Esta técnica terapêutica visa não apenas tratar a região afetada do corpo, mas sim agir sobre o sistema nervoso como um todo, promovendo o sistema de equilíbrio e compensação em todo o corpo (Alvarenga et al., 2014). Os acupontos são tidos na Medicina Tradicional Chinesa, como os locais mais externos do corpo energético de um indivíduo, trabalhando como um vínculo de comunicação entre o meio externo e o meio interno (Alvarenga et al., 2014; Gil, 2019; Grillo, 2011; Oliveira, 2020; Rando-Meirelles, Gonçalo, \& Sousa, 2009; Sousa, 2018). Através da incitação destes pontos é possível alterar a movimentação da circulação sanguínea além de realizar o relaxamento muscular, encerrando espasmos e reduzindo a dor e a inflamação, também é possível gerar a liberação de hormônios como as endorfinas e o cortisol gerando a analgesia (Alvarenga et al., 2014; Gil, 2019; Grillo, 2011; Oliveira, 2020; Sousa, 2018).

A acupuntura é considerada uma das mais antigas e respeitadas ciências de todo o mundo, em especial pelos orientais mais especificamente na china, local onde tal ciência iniciou sua disseminação como segredo de família (Alvarenga et al., 2014; Boleta-Ceranto \& Miura, 2013; Sousa, 2018). A acupuntura vem sendo usada a mais de cinco mil anos pelos chineses, sendo que atualmente existem mais de 3 mil anos de registros escrito e mais de 2 mil anos de achados arqueológicos tais como agulhas feitas de pedras, inscrições em osso e carapaças de animais, tal fato comprova que este procedimento teve uma longa trajetória de evolução (Alvarenga et al., 2014; Boleta-Ceranto \& Miura, 2013; Sousa, 2018).

Está ciência se fundamente filosoficamente no conceito de Ying - Yang e na teoria dos cinco elementos (BoletaCeranto \& Miura, 2013; Gil, 2019). O Ying - Yang se refere as duas partes opostas e complementares da natureza, os quais se relacionam reciprocamente, tal ideia representa tanto dois elementos opostos, bem como dois fatores que formam a essência de um aspecto (Boleta-Ceranto \& Miura, 2013; Gil, 2019). Seguindo esta filosofia, Ying representa qualidades negativas, enquanto Yang representa as positivas, sendo que um não consegue existir sem o outro, logo pode-se se concluir que não existe nem Ying nem Yang absolutos (Boleta-Ceranto \& Miura, 2013; Gil, 2019; Sousa, 2018) A união de Ying e Yang formam Tai Chi que é o princípio e o fim, ou seja, a vida e a morte, de acordo com esta crença tais condições opostas e complementares 
devem manter um perfeito equilíbrio (Boleta-Ceranto \& Miura, 2013; Gil, 2019; Sousa, 2018).

Já na teoria dos cinco elementos, nos primórdios, os chineses perceberam através da pratica e observação da vida, entre diversas coisas, que fogo, madeira, metal, terra e água são essenciais para a constituição da natureza (Boleta-Ceranto \& Miura, 2013; Sousa, 2018). Através da imersão dos chineses nos conhecimentos materiais, criaram a teoria dos cinco elementos da natureza, bem como suas relações, atividades e mudanças, que ocorriam entre estes, um gera o outro, ou inibi ou até mesmo gera uma contra inibição, esta denominada de vinculo mãe-filho, na qual também é necessário a manutenção de um equilíbrio, posteriormente transpassaram essa relação para o desenvolvimento das doenças (Boleta-Ceranto \& Miura, 2013; Sousa, 2018).

A acupuntura é uma forma terapêutica com destaque no diagnóstico individualizado, geração de conexão terapêutica e integração do indivíduo com o meio ambiente, gerando um estimulo ao autocuidado (Sousa, 2018). Esta vem se mostrando como uma terapêutica integrativa, complementar e alternativa extremamente eficiente dentro da odontologia, partindo dos princípios de integração, união e solidificação de todos os conhecimentos técnicos odontológicos e científicos com agregação do grande conhecimento trazido pela Medicina Tradicional Chinesa, de tal modo obtendo os melhores resultados possíveis para o paciente, bem como uma qualidade de vida melhor e um maior bem estar (Alvarenga et al., 2014; Grillo, 2011; Rando Meirelles et al., 2009; Sousa, 2018).

Nos últimos anos a acupuntura assim como toda a Medicina Tradicional Chinesa vem recebendo um número gigantesco de nos adeptos, tanto adeptos que a praticam quanto aquele que se submetem a seu tratamento (Grillo, 2011; Sousa, 2018). A acupuntura vem chamando uma atenção especial dentro da odontologia devido a suas propriedades analgésicas, antiinflamatórias, ansiolíticas, mio relaxantes e ativadora da função imune, tal técnica vem demostrando resultados extremamente satisfatórios quando associada a uma série de procedimentos odontológicos (Grillo, 2011; Rando-Meirelles et al., 2009; Sousa, 2018; Zotelli et al., 2010).

Na porção ocidental do mundo a acupuntura começou a ser estudada e teve sua eficácia comprovada apenas após 1970, sendo que os primeiros estudos realizados dentro da área odontológicas sugiram em 1974 na França sendo realizados pelo Dr. Michel Bresset, o qual adquiriu conhecimento a cerca desta terapia em uma de suas viagens a China e desde então a acupuntura vem sendo cada vez mais utilizada dentro dos consultórios odontológicos (Alvarenga et al., 2014; Grillo, 2011; Taffarel \& Freitas, 2009; Sousa, 2018).

Atualmente, a Organização Mundial da Saúde (OMS) incentiva a evolução da acupuntura tanto em nível investigativo quanto prático (Sousa, 2018). Países como Portugal está entre a dezena de países da União Europeia que já possui legislação de integração de Medicinas Complementares Alternativas (MCAs), tais como a acupuntura, porem até este momento apenas os profissionais médicos devidamente qualificados se encontram habilitados a exerce-las (Sousa, 2018).

Estudos realizado com pacientes que foram submetidos a procedimentos cirúrgicos para a extração de terceiros molares mandibulares e maxilares, os quais também se submeteram a acupuntura como terapêutica alternativa à terapêutica farmacológica convencional, apresentaram redução da sintomatologia dolorosa no pós operatório (Arslan et al., 2019; Gil, 2019; Gil et al., 2020).

Em outros diversos estudos, o uso da acupuntura beneficiou os pacientes e os profissionais, em todas as etapas dos procedimentos cirúrgicos para extração de terceiros molares, uma vez que esta técnica se mostrou eficiente auxiliando o paciente no controle da ansiedade nos momentos pré e transoperatórios, também favoreceu o profissional e o paciente no controle de sangramento durante o procedimento cirúrgico e ainda beneficiou o paciente no momento pós operatório reduzindo a intensidade e a duração da dor, amenizando o surgimento de inflamação e edemas e consequentemente diminuindo a quantidade necessária de medicamentos no pós-cirúrgico o que proporcionou uma grande proteção para paciente frente aos diversos efeitos colaterais causados pelos medicamentos utilizados nesta fase do tratamento (Alvarenga et al, 2014; Gil et al., 
2020; Gil, 2019; Linde, Niemann \& Meissner, 2010; Armond, 2017).

\section{Discussão}

Os estudos de Boleta-Ceranto e Miura (2013), assim como os estudos de Grillo (2011) e de Arslan et al. (2019), demonstraram que cerca de $80 \%$ dos pacientes que foram submetidos a terapia de acupuntura, obtiveram resultados positivos apresentando redução significativa da dor pós operatória, após procedimento odontológico, o que por sua vez reduziu a quantidade de analgésicos consumidos pelo paciente no pós operatório, deixando-o menos exposto a possíveis efeitos colaterais desses fármacos.

No estudo de Arslan et al. (2019), é exposto alguns efeitos colaterais da acupuntura, sendo os mais leves, fadiga, dor, sonolência e sangramento e os mais graves sendo endocardites e hepatite, porem o mesmo explica que na grande maioria das vezes, tais efeitos ocorrem por ignorância da anatomia básica e/ou falta de protocolos assépticos, ambos trazem risco para os pacientes porem este último traz ainda o agravante da possibilidade de ocorrer infecções cruzadas.

Lindea (2010) traz um achado no mínimo curioso, em seu estudo é demonstrado que placebos de acupuntura são mais eficientes que os placebos farmacológicos.

Michalek-Sauberer (2012) comprovou que, a acupuntura auricular pode ser utilizada, como método preventivo para pacientes agendados, que solicitarem uma intervenção prévia ao procedimento odontológico, para reduzir seus níveis de ansiedade. Também foi demonstrado em seu estudo, que pacientes do sexo feminino demonstravam maiores níveis de ansiedade, antes da realização da acupuntura. Também foi demonstrado no trabalho de Michalek-Sauberer (2012), que o fato do paciente crer ou não na acupuntura, não afeta no resultado da terapia.

Nos estudos de Gil (2019, 2020), foi comprovado que o uso da acupuntura auxilia na redução do sangramento, durante o procedimento de exodontia de terceiros molares, o que por sua vez, melhora a visão do campo operatório, permitindo que o cirurgião dentista consiga realizar um procedimento mais preciso, gerando mais conforto para seu paciente. Gil (2019, 2020), também comprovou que, a acupuntura melhorou o pré e o pós-operatório, após a remoção de terceiros molares reduzindo a ansiedade, o sangramento, a dor e o edema.

No trabalho de Gil (2020), também foi demonstrado que, apesar do uso da acupuntura, não houve diferença no tempo do procedimento cirúrgico e nem na quantidade de anestésico que foi utilizado, porém, mesmo assim, os resultados proporcionados pela acupuntura foram excelentes, uma vez que, reduziu a ansiedade do paciente, auxiliou o profissional e o paciente reduzindo o volume de sangramento, proporcionou maior conforto para o paciente, uma vez que reduziu a dor e o edema pós operatório e também forneceu uma maior segurança e proteção para o paciente, já que, este necessitou de uma menor quantidade de medicamentos no pré-operatório para combater a ansiedade e no pós-operatório para combater a dor e o edema, o que acabou expondo o paciente, a um menor risco de efeitos colaterais dos fármacos.

\section{Conclusão}

Após analisar todo o material bibliográfico de referência, concluiu-se que, a acupuntura, apesar de ser uma técnica milenar e ao mesmo tempo tão recente no ocidente, ainda mais dentro da área odontológica, podendo ser usada em todos os procedimentos clínicos, principalmente, nas cirurgias odontológicas tanto no pré-operatório, no transoperatório como também no pós-operatório, proporcionando conforto e segurança, tanto para o profissional, quanto para o paciente.

Sugerimos que nos trabalhos futuros sejam realizados estudos com grupos amostrais maiores para que os resultados obtidos possam ser melhor analisados e em maior escala, facilitando uma melhor compreensão acerca desta técnica. 


\section{Referências}

Alvarenga, T. F., Amaral, C. G., \& Steffen, C. P. (2014). Ação da acupuntura na neurofisiologia da dor: revisão bibliográfica. Rev Amaz Sci Health, 2(4), 2936. http://www.ojs.unirg.edu.br/index.php/2/article/view/530

Armond, A. C. V. (2017). Acupuntura no controle de dor, edema, trismo e ansiedade associados à exodontia de terceiros molares mandibulares: ensaio clínico randomizado controlado triplo cego. Dissertação de Mestrado em Odontologia, Universidade Federal dos Vales do Jequitinhonha e Mucuri, Diamantina, MG.

Armond, A. C. V., Glória, J. C. R., Santos, C. R. R., Galo, R., \& Falci, S. G. M. (2019). Acupuncture on anxiety and inflammatory events following surgery of mandibular third molars: a split-mouth, randomized, triple-blind clinical trial. Int J Oral Maxillofac Surg, 48(2), 274-281. doi.org/10.1016/j.ijom.2018.07.016

Arslan, H., Ahmed, H. M. A., Yıldız, E. D., Gündoğdu, E. C., Seçkin, F., \& Arslan, S. (2019). Acupuncture reduces the postoperative pain in teeth with symptomatic apical periodontitis: a preliminary randomized placebo-controlled prospective clinical trial. Quintessence Int Stud, 50(4), 270-277. 10.3290/j.qi.a42153

Boleta-Ceranto, D. C. F., \& Miura, C. S. N. (2013). Analgesia por Acupuntura na Odontologia. In: S. Silvério-Lopes (ed.). Analgesia por Acupuntura (pp. 93106). Curitiba: Omnipax. 10.7436/2013.anac.07

Brum, C. N. et al. (2015). Revisão narrativa da literatura: aspectos conceituais e metodológicos na construção do conhecimento da enfermagem. In: Lacerda, M. R., \& Costenaro, R. G. S. Metodologia de pesquisa para a enfermagem: a saúde da teoria a prática. (pp. 123-142).

Chiapasco, M., Cicco, L., \& Marrone, G. (1993). Side effects and complications associated with third molar surgery. Oral Surg Oral Med Oral Pathol, 76(4), 412-420. doi.org/10.1016/0030-4220(93)90005-O

Florian, M. R., Rando-Meirelles, M. P. M., \& Sousa, M. L. R. (2012). Uso da acupuntura em um caso de parestesia dos nervos alveolares inferior e lingual. Rev. Assoc. Paul. Cir. 66(4), 312-315. Disponível em: http://revodonto.bvsalud.org/scielo.php?pid=S0004-52762012000400011\&script=sci_arttext

Gil, M. L. B., Marinho, L. M. R. F., Moraes, M., Wada, R. S., Grappo, F. C., \& Sato, J. et al. (2020). Effectiveness of Acupuncture in Dental Surgery: A Randomized, Crossover, Controlled Trial. J Acupunct Meridian Stud, 13(3), 104-109. doi.org/10.1016/j.jams.2020.03.063

Gil, M. L. B. (2019). Efeito da regulação energética com acupuntura em exodontia de terceiros molares. Tese de Doutorado em Odontologia, Universidade de Campinas, Piracicaba, SP. 2019. http://repositorio.unicamp.br/handle/REPOSIP/335712

Grillo, C. M. (2011). Efeito da acupuntura no manejo da dor aguda de origem dental. Dissertação de Mestrado em Odontologia, Universidade Estadual de Campinas, Piracicaba, SP. http://repositorio.unicamp.br/handle/REPOSIP/288045

Günther, H. (2006). Pesquisa qualitativa versus pesquisa quantitativa: esta é a questão? Psicologia: teoria e pesquisa, 22(2), 201-210. https://www.scielo.br/pdf/ptp/v22n2/a10v22n2.pdf

Linde, K., Niemann, K., \& Meissner, K. (2010). Are sham acupunture interventions more effective tha (other) placebos? A re-analysis of data from the cochrane review on placebo effects. Rev Karger GmbH. 17(5), 259-264. doi.org/10.1159/000320374

Lux, E. A., Wahl, G., Erlenwein, J., Wiese, C., \& Wirz, S. (2017). Ist die Supplementierung der ohrakupunktur bei operativer Zahnentfernung in Lokalanästhesie effektiv? Rev Sprin Med Verl GmbH, 31, 489-498. https://link.springer.com/article/10.1007/s00482-017-0212-3

Michalek-Sauberer, A., Gusenleitner, E., Gleiss, A., Tepper, G., \& Deusch, E. (2012). Auricular acupuncture effectively reduces state anxiety before dental treatment--a randomised controlled trial. Clin Oral Investig, 16(6), 1517-1522. https://link.springer.com/article/10.1007\%2Fs00784-011-0662-4

Oliveira, S. J. (2020). O uso da acupuntura como terapia complementar na odontologia. Trabalho de Conclusão de Curso de Odontologia, Universidade UNIFACVEST. ttps://www.unifacvest.edu.br/assets/uploads/files/arquivos/28526-oliveira,-s.-j.-o-uso-sa-acupuntura-como-terapia-complementar-naodontologia.-odontologia.-lages_-unifacvest,-2020-01_.pdf

Pereira, A. S., Shitsuka, D. M., Parreira, F. J., \& Shitsuka, R. (2018). Metodologia da pesquisa científica. UFSM.

Rando-Meirelles, M. P. M. R., Gonçalo, C. S., \& Sousa, M. L. R. (2009). Manejo da dor orofacial através do tratamento com acupuntura: relato de um caso. Rev. odontol. UNESP (Online), 38(6):379-382. https://webzoom.freewebs.com/citeacupuntura/documents/atm\%20II.pdf

Santana-Santos, T., Souza-Santos, J. A. S., Martins Filho, P. R. S., Silva, L. C. F., Silva, E. D. O., \& Gomes, A. C. A. (2013). Prediction of postoperative facial swelling, pain and trismus following third molar surgery based on preoperative variables. Med Oral Patol Oral Cir Bucal, 18(1), 65-70. doi.org/10.4317\%2Fmedoral.18039

Santos, T. L., Santos, E. J. L., Lins, R. B. E., Araújo, L. F., Mesquita, B. S., \& Sobreira, T. (2014). Qualidade de vida de pacientes submetidos à exodontia de terceiros molares. Rev Odontol UNESP, 44(1), 6-11. doi.org/10.1590/1807-2577.1055

Sousa, A. A. (2018). Acupuntura no tratamento médico-dentário. Dissertação de Mestrado em Medicina Dentária, Universidade Fernando Pessoa, Porto, Portugal. https://bdigital.ufp.pt/handle/10284/7286

Taffarel, M. O., \& Freitas, P. M. C. (2009). Acupuntura e analgesia: aplicações clínicas e principais acupontos. Cienc. Rural, 39(9), 2665-2672. doi.org/10.1590/S0103-84782009000900047

Zotelli, V. L. R., Meirelles, M. P. M. C., \& Sousa, M. L. R. (2010). Uso da acupuntura no manejo da dor em pacientes com alterações na articulação temporomandibular (ATM). Rev. odontol. Sniv. Cid. $22(2), 185-188$. http://arquivos.cruzeirodosuleducacional.edu.br/principal/old/revista_odontologia/pdf/maio_agosto_2010/unicid_22_02.pdf\#page=97 APA. 